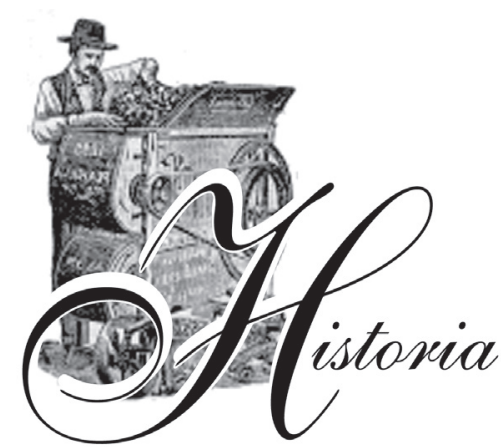

de la Ciencia en México
Recepción: 11 de marzo de 2016

Aceptación: 21 de diciembre de 2016

*University of Bristol, United Kingdom. Correo electrónico: jorge.herreraflores@bristol.ac.uk Se agradecen los comentarios de los árbitros de la revista y a la doctora Celine Armenta Olvera, doctora María Eugenia Cabrera Bruschetta, doctor Jean Brunet, licenciado Oscar A. Irala Álvarez y el arquitecto Enrique Martínez Molina por la valiosa información proporcionada. Asimismo, al Archivo General del Estado de Puebla (AGEP) por la facilidad otorgada para acceder a su acervo hemerográfico. Se reconoce especialmente al señor Moisés Cabrera Huerta por la entrevista concedida e información inédita aportada.

(c)

\section{Introducción}

El Valle de Puebla concentra importantes localidades fosilíferas del Pleistoceno, en las cuales han dado notables hallazgos que en su mayoría se concentran en la zona de Valsequillo. Los primeros trabajos paleontológicos para el Valle de Puebla datan de finales del siglo XIx, cuando el maestro José Manzo y Jaramillo creó la colección de fósiles del Gabinete de Historia Natural del Colegio del Estado. A finales de

\title{
Investigaciones paleontológicas en el Valle de Puebla durante el siglo xx
}

\author{
Jorge A. Herrera Flores*
}

Resumen. Se ofrece una extensa revisión bibliográfica y hemerográfica de los trabajos paleontológicos realizados en el Valle de Puebla desde principios del siglo xx hasta la actualidad. Se hace especial énfasis en los estudios efectuados por el Proyecto Valsequillo y la Fundación Alemana para la Investigación Científica, dado que estos aportaron las mayores contribuciones al conocimiento de la paleontología del Valle de Puebla. Además, se proporcionan datos inéditos sobre las exploraciones que dieron origen a la colección paleontológica del extinto Museo de Historia Natural de Puebla y se comenta sobre el inicio de las investigaciones actuales y su perspectiva hacia el futuro.

Palabras clave: fósiles, paleontología, Pleistoceno, Puebla, Valsequillo.

\section{Paleontological Research in the Valley of Puebla During the 20th Century}

Abstract. The present work offers a bibliographical and hemerographic review of the paleontological works carried out in the Valley of Puebla since the beginning of the 20th century to the present day. Special emphasis is placed on the studies carried out by the Valsequillo Project and the German Research Foundation, because of the huge contributions that both projects provided to the current knowledge of the paleontology of the Valley of Puebla. In addition, unpublished data on the explorations that originated the paleontological collection of the extinct Museum of Natural History of Puebla are provided. This work also discusses the beginning of current research and its perspective towards the future.

Keywords: fossils, paleontology, Pleistocene, Puebla, Valsequillo.

ese siglo en 1891, los geólogos Félix y Lenk publicaron un estudio sobre la geología y paleontología de México en el que se incluyó al estado de Puebla (Cruz-Muñoz, 2001; Rojas-Cortés, 2004, 2016). Sin embargo, los descubrimientos e investigaciones científicas más notables ocurrieron a lo largo del siguiente siglo, gracias a que renombrados paleontólogos e instituciones de gran prestigio enfocaron sus estudios en la fauna que habitó esta zona durante el Pleistoceno.
$\mathrm{Al}$ presente, las investigaciones sobre la extinta fauna del Valle de Puebla continúan, pese a las complicaciones para realizar nuevas prospecciones dado el continuo crecimiento de la mancha urbana de la ciudad de Puebla. No obstante el nuevo material colectado, así como el aún disponible del Proyecto Valsequillo y otras prospecciones anteriores, aún provee valiosa información sobre la prehistoria de Puebla. A continuación se ofrece un recuento de las investigaciones paleontológicas más 
importantes llevadas a cabo a lo largo del siglo $\mathrm{xx}$.

\section{Los inicios de un siglo muy productivo}

A inicios del siglo xx el renombrado paleontólogo Henry Fairfield Osborn visitó el Valle de Puebla para extraer material paleontológico de la zona de Valsequillo y estudiarlo en los Estados Unidos de América (Armenta-Camacho, 1978; Rojas-Cortés, 2016). Posteriormente, Osborn (1905) publicó un breve artículo en el que menciona la presencia de Elephas imperator (Mammuthus columbi), así como otros restos de mamíferos provenientes de la localidad de Totime-

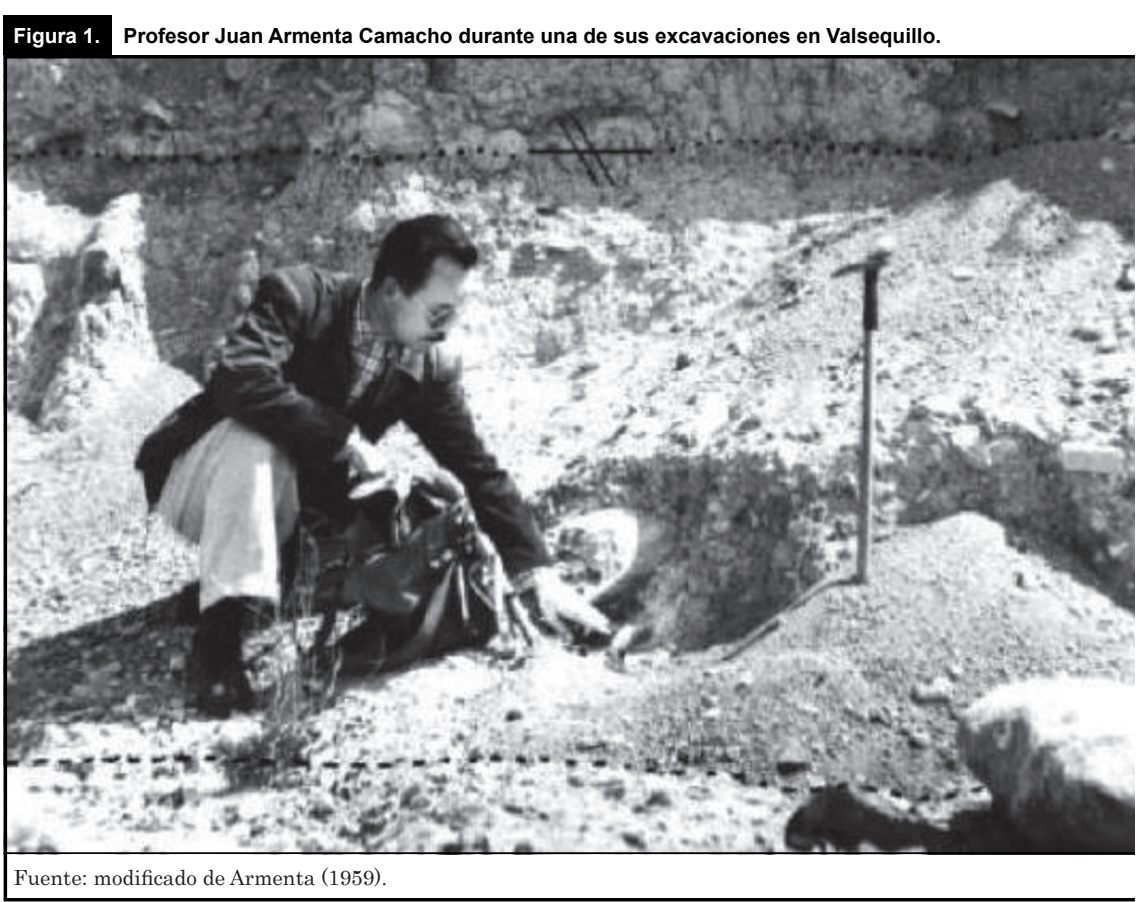

Figura 2. Nota periodística con el anuncio de los hallazgos del profesor Juan Armenta.

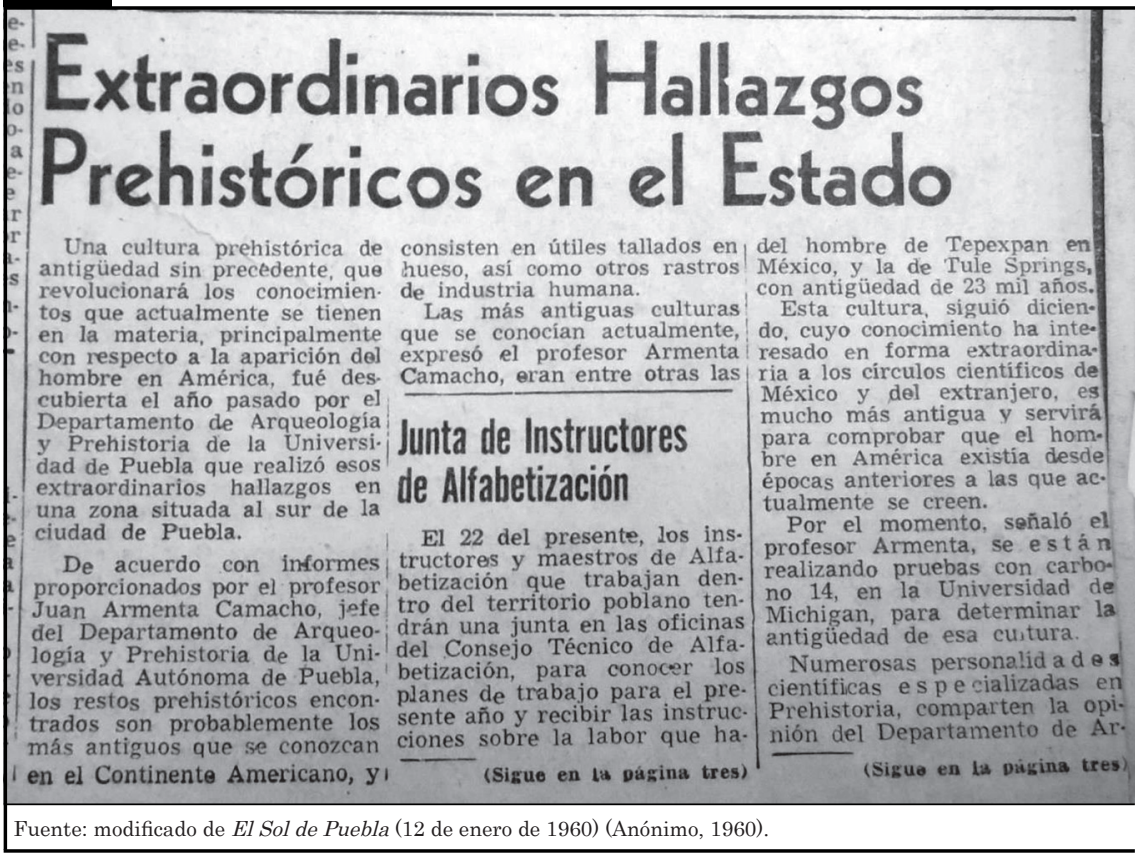

huacán, los cuales se perdieron debido a un incendio en su trayecto a los Estados Unidos. Tiempo después, el investigador alemán Wilhelm Freudenberg publicó en 1910 y en 1922 los resultados de sus investigaciones sobre la fauna pliocénica y pleistocénica de México. Freudenberg también destacó la fauna de Valsequillo. Valga como ilustración, que registró los géneros: Antilocapra, Canis, Capromeryx, Cervus, Cuvieronius, Equus, Felis, Hyaenognathus (Borophagus), Mammuthus, Mastodon (Mammut), Procamelus, Prosthennops, Tapirus, Urocyon y Ursus (Miller y Carranza-Castañeda, 1984; Rojas-Cortés, 2016). Durante la década de los cincuenta, el antropólogo Mario Pichardo elaboró una detallada revisión de los proboscídeos fósiles de México y en 1960 publicó su investigación en la que menciona para Valsequillo a los géneros Cuvieronius y Mammuthus (Pichardo del Barrio, 1960).

\section{El Proyecto Valsequillo}

El profesor Juan Armenta Camacho, catedrático de la Universidad Autónoma de Puebla, enfocó la mayor parte de su carrera en realizar estudios sobre prehistoria (figura 1).

Su interés en los fósiles surgió en junio de 1933 cuando descubrió restos de un mamut en el cauce del río Alseseca, lugar en el que dos años más tarde encontró el fémur de un proboscídeo con un objeto de pedernal enclavado (Armenta-Camacho, 1978). Tras varios años de investigar los mamíferos fósiles de Valsequillo, Armenta (1959) publicó una breve monografía en la que dio a conocer un artefacto tallado asociado a huesos de mamut imperial (Mammuthus columbi) (Agenbroad, 2005). En 1960 el profesor Armenta dio a conocer a los medios de comunicación sus importantes hallazgos (figura 2), los que llamaron la atención de investigadores extranjeros (Anónimo, 1960). 
Uno de los primeros interesados en conocer más sobre estos hallazgos, fue la doctora Cynthia Irwin-Williams de la Universidad de Harvard, quien en 1960 visitó Valsequillo con el profesor Armenta, y desde ahí comenzó un acuerdo de colaboración entre ambos y sus respectivas instituciones (IrwinWilliams, 1962).

Un año más tarde, la doctora IrwinWilliams y el profesor Armenta obtuvieron una beca de la American Philosophical Society con la que comenzaron en 1962 el Proyecto Valsequillo (Irwin-Williams, 1962; Irwin-Williams y Armenta-Camacho, 1963). Para 1963, presentaron ante la American Philosophical Society su reporte de actividades en el que informaron su trabajo en cuatro localidades: El Mirador, Hueyatlaco, Tecacaxco y El Horno, en donde encontraron diversos artefactos de origen humano asociados con la fauna extinta de la zona. En 1964 la doctora Irwin-Williams y el profesor Armenta dieron comienzo a nuevos trabajos de campo, gracias al apoyo económico de la National Science Foundation. Además, se integraron al proyecto los doctores Harol E. Malde del U. s. Geological Survey y Clayton Ray del Smithsonian Institution, quienes condujeron los estudios de geología y paleontología respectivamente (Irwin-Williams, 1964). En 1966 comenzó la última etapa de campo del Proyecto Valsequillo, a la que se integró la entonces estudiante de posgrado Virginia Steen-McIntyre. Esta etapa principalmente se enfocó en la localidad de Hueyatlaco, en la que se buscaron restos de fauna y materiales de culturas humanas, además de un completo estudio geológico y paleontológico de la zona (Irwin-Williams, 1966).

Ya para 1967 inició la polémica que envuelve hasta el día de hoy al Proyecto Valsequillo y a sus integrantes. Cuando la doctora Irwin-Williams publicó los resultados preliminares sobre los objetos de culturas humanas prehistóricas asociadas a restos de caballos, camellos y mastodontes de Valsequillo (Irwin-Williams, 1967). El doctor José Luis Lorenzo, entonces director del Departamento de Prehistoria del Instituto Nacional de Antropología e Historia manifestó que los artefactos humanos asociados a los fósiles pudieron haber sido implantados en el sitio por algunos de los trabajadores de la excavación (Lorenzo, 1967). Por su parte, la doctora Irwin-Williams respondió que las acusaciones del doctor Lorenzo eran malintencionadas e infundadas y en 1969 publicó una nota en la que ofrecía el testimonio de tres renombrados arqueólogos que reafirmaban la validez de los hallazgos (Irwin-Williams, 1969). No obstante, la polémica se acrecentó en 1969 cuando los doctores Barney J. Szabo, Harold E. Malde y Cynthia Irwin-Williams publicaron un artículo donde dieron a conocer que mediante nuevas técnicas de datación, los artefactos encontrados en Valsequillo ofrecían una edad superior a los 200000 años, edad fuera de toda proporción respecto a lo aceptado por la comunidad científica sobre la antigüedad del hombre temprano en América. Sin embargo, los investigadores atribuyeron esta anomalía a lo reciente de las nuevas técnicas ya que aún estaban en fase experimental (Szabo et al., 1969).

Todo lo anterior desató conflictos entre los integrantes del Proyecto Valsequillo, quienes en su mayoría dejaron de cooperar entre sí. Debido a este motivo y tras varios conflictos, fue hasta 1978 que el profesor Juan Armenta publicó un libro en que dio a conocer los detalles de sus investigaciones paleontológicas. En este, se informó principalmente sobre los huesos con labor humana, con los que efectuó diversos experimentos para comprobar su autenticidad (ArmentaCamacho, 1978). En ese mismo año la doctora Irwin-Williams publicó un capítulo de libro en el que dio detalles de las investigaciones y mencionó que las dataciones por radiocarbono ofrecían más de 20000 años de antigüedad para los fósiles y objetos humanos, edad que, aunque considerablemente más reciente que la de sus estudios anteriores, no dejaba de ser controversial. Asimismo, la doctora Irwin-Williams criticó los estudios de datación hechos por el grupo de la doctora Steen-McIntyre por emplear métodos no confiables, que arrojaban datos anómalos y no conclusivos (Irwin-Williams, 1978). En 1981, los doctores Virginia SteenMcIntyre, Harold E. Malde y Roald Fryxell publicaron un artículo en el que indicaron una edad de más de 250000 años para los restos fósiles y objetos humanos asociados, lo que provocó una respuesta de parte de la doctora Irwin-Williams en la que desestimó que los restos de la zona pudieran tener tal antigüedad (Steen-McIntyre et al. 1981; Irwin-Williams, 1981). Finalmente, en 1990 falleció la doctora Irwin-Williams sin que los resultados finales y conclusiones de sus investigaciones salieran a la luz.

\section{La Fundación Alemana para la Investigación Científica}

A mediados de la década de los sesenta, de manera totalmente independiente al Proyecto Valsequillo, comenzaron una serie de investigaciones MéxicoAlemania patrocinadas por la Fundación Alemana para la Investigación Científica mediante el denominado Proyecto México. Estas investigaciones abarcaban una amplia gama de disciplinas tales como arqueología, botánica, etnología, geografía, geología, historia y paleontología encauzadas al estudio de la región Puebla-Tlaxcala 
(Treue, 1968). Correspondiente a la paleontología, los estudios empezaron en 1965 por el doctor Ekke W. Guenther de la Universidad de Kiel (figura 3), quien se enfocó en la zona de Valsequillo para llevar a cabo diversos trabajos geológicos y paleontológicos como el estudio de los sedimentos de la zona, o colecta y clasificación taxonómica de vertebrados del Pleistoceno, además de comparar los fósiles con los recolectados en Estados Unidos y Europa (Guenther, 1968; Guenther y Bunde, 1973). Guenther $(1967,1968)$ publicó un reporte preliminar sobre la geología de Valsequillo y un año más tarde un listado también preliminar de la fauna fósil. Entre los taxones reportados se hallaban los géneros Testudo (Testudines), Canis y Smilodon (Carnivora), Antilocapra, Cervus, Sus, Bison, Camelus (Artiodactyla), Mastodon, Mammuthus, Parelephas, Archidiskodon (Proboscidea), Glyptodon y Megatherium (Edentata), así como diversos roedores y perisodáctilos.

Los últimos trabajos paleontológicos del Proyecto México tuvieron lugar en 1968 por parte del doctor
Horst Bunde, mientras que en 1973 se publicó un libro que incluía los resultados de las investigaciones geológicas y paleontológicas de la Fundación Alemana para la Investigación Científica. Guenther (1973a) y Guenther y Bunde (1973) señalan que los restos fósiles poseen una edad aproximada de 26000 años según las dataciones con radiocarbono hechas en la Universidad de Kiel, además ofrecen el listado de las especies encontradas durante sus exploraciones: Canis latrans, Canis dirus, Smilodon gracilis, Mammuthus columbi, Mammuthus imperator, Mammuthus primigenius, Equus of. E. mexicanus, Equus cf. E. conversidens, Platygonus compressus alemanii, Camelops of. C. minidokae, Camelops hesternus, Tanupolama stevensi, Breameryx mexicana, Stockoceros conklingi, Tetrameryx of. T. shuleri, Bison sp., Holmesia septentrionalis, Brachyostracon cylindricus, Nothrotherium of. N. shastense. Cabe señalar que algunos de estos géneros o especies están en desuso o son sinónimos de otros, por lo que la discusión sobre su actual y correcta clasificación taxonómica va más allá del objetivo de este trabajo.

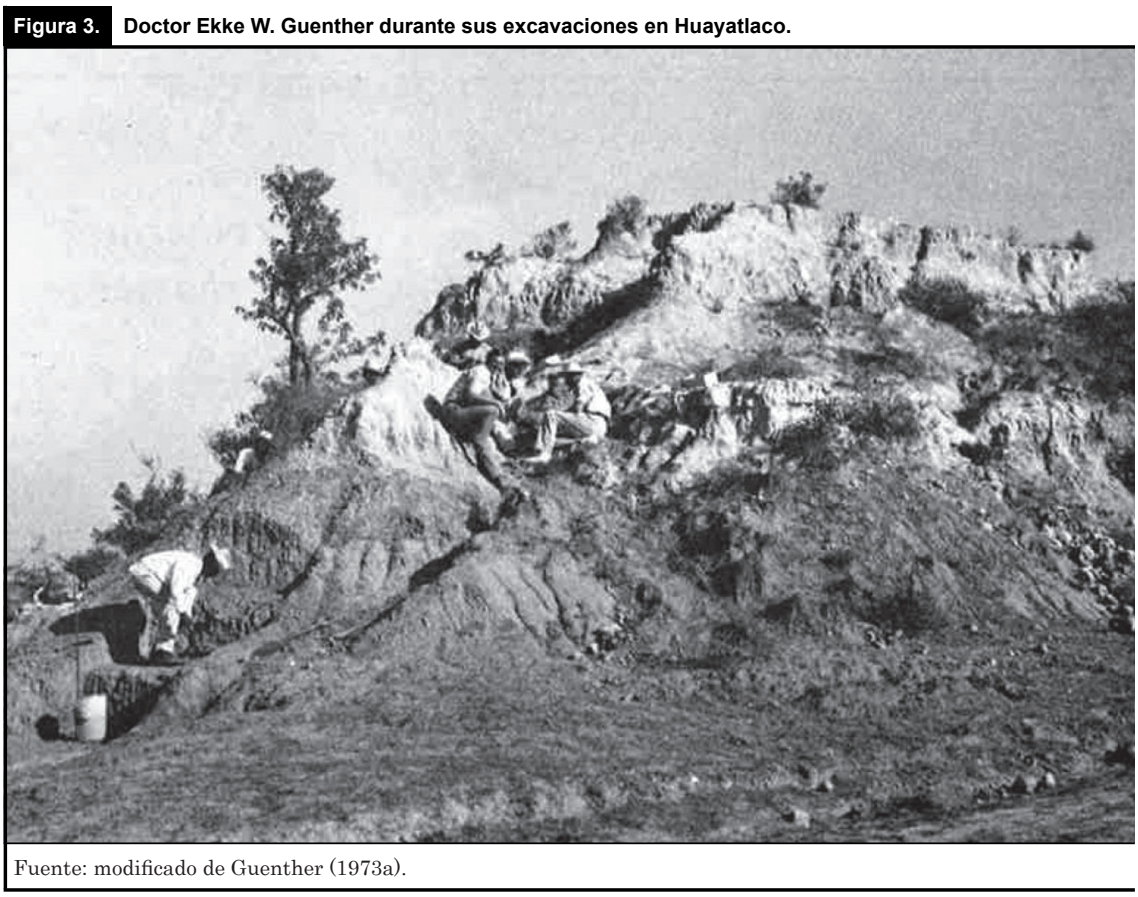

Dentro de los resultados finales, Bunde (1973) ofreció un estudio detallado de la geología de Valsequillo en el que menciona que los depósitos de la zona van desde el Albiano (Cretácico Inferior) hasta el Reciente. Nobis (1973) reportó una gran cantidad de dientes aislados de équidos fósiles, provenientes de cinco localidades: Hueyatlaco, San Antonio Arenillas, Atepetzingo, Caulapan y Zacachimalpa, e identificó que pertenecían a las especies Equus mexicanus, Equus conversidens y a una especie no identificada. Por su parte, Guenther (1973b) reportó varios molares aislados de proboscídeos correspondientes a cuatro especies: Mammuthus columbi, Mammuthus imperator, así como dos especies no determinadas; no obstante, $M$. imperator es en la actualidad sinónimo de M. columbi (Agenbroad, 2005). Es importante señalar que aunque los investigadores de la Fundación Alemana para la Investigación Científica trabajaron prácticamente en las mismas localidades que el Proyecto Valsequillo, no reportaron indicios de culturas humanas asociados a la fauna fósil de la región.

\section{Moisés Cabrera Huerta y el Museo de Historia Natural de Puebla}

Tras la finalización del Proyecto Valsequillo y del Proyecto México, pasaron varios años para que surgieran nuevas investigaciones paleontológicas en el Valle de Puebla. Sin embargo, durante un largo tiempo se llevaron a cabo de forma amateur una serie exploraciones y el rescate de fósiles en Valsequillo por parte del señor Moisés Cabrera Huerta. Su historia, aunque casi desconocida, es la de uno de los mejores paleontólogos amateurs de México. Su trabajo comenzó de forma autodidacta cuando de joven se mudó de Veracruz a Puebla, y así dio inicio a la colecta de fósiles en 
los alrededores del lago de Valsequillo. Posteriormente, durante la década de los sesenta, Moisés Cabrera Huerta contactó al profesor Juan Armenta a quien informó sobre su afición por la paleontología y le ofreció su ayuda para colectar fósiles en Valsequillo (figura 4).

Por medio del profesor Armenta, Moisés Cabrera Huerta tuvo contacto con algunos investigadores extranjeros, quienes se interesaron en sus hallazgos de los que destacaba el esqueleto casi completo de un gliptodonte juvenil (Cabrera Huerta, comunicación personal, 2016).

Hacia finales de la década de los setenta, Moisés Cabrera Huerta contaba con una amplia colección de fósiles de Valsequillo, que contempló donar al Museo de Historia Natural de la Ciudad de México o al Museo Nacional de Antropología, pero al enterarse de la próxima creación del Museo de Historia Natural de Puebla optó por entregarla a este nuevo recinto, con el fin de que los fósiles permanecieran en el estado de Puebla (Cabrera Huerta, comunicación personal, 2016). Una vez terminado el Museo de Historia Natural de Puebla, el personal y Moisés Cabrera Huerta emprendieron diversas exploraciones en la zona de Valsequillo con el objetivo de rescatar material paleontológico para resguardarlo y exhibirlo. De las distintas exploraciones realizadas, se rescataron un importante número de fósiles, principalmente húmeros, fémures, defensas, mandíbulas completas y molares de mamut (Mammuthus sp.) y mastodonte (Mammut sp.), cráneos incompletos de bisonte (Bison sp.), mandíbulas y dientes aislados de caballo (Equus sp.) y camello (Camelops sp.), los cuales fueron instalados en grandes vitrinas de la Sala de Prehistoria (HerreraFlores, 2015). Dentro de la colección expuesta, destacaban un par de defensas de proboscídeo localizadas a las orillas del lago de Valsequillo, las cuales son de las más grandes y completas halladas en el estado de Puebla: medían más de $2.5 \mathrm{~m}$ de longitud. Cabe destacar que las exploraciones paleontológicas en Valsequillo y en otras zonas del estado de Puebla fueron constantes por parte del Museo de Historia Natural de Puebla hasta que cerró sus puertas en diciembre del 2002. Tras el cierre de este museo, pocos fósiles se conservaron en el Museo Imagina, recinto que ocupó su lugar (el Museo Imagina recientemente se remodeló y se renombró Museo de la Evolución), mientras que algunos especímenes se entregaron al centro INAH-Puebla. Aún así, se desconoce el paradero actual de la mayoría de los fósiles que estuvieron alojados en el extinto Museo de Historia Natural de Puebla (observación personal del autor, 2016).

\section{Investigaciones contemporáneas}

Hacia finales del siglo pasado, nuevas investigaciones comenzaron a desarrollarse, entre las que destacó el rescate de restos de mamut en la colonia Tres
Cerritos, al sur de la ciudad de Puebla. El hallazgo tuvo su origen cuando una persona avisó al INAH-Puebla sobre la presencia de fósiles en los alrededores de su domicilio (Lagunas-Rodríguez y Suárez-Cruz, 1997), por lo que investigadores del instituto procedieron a su rescate y estudio. Como resultado de esta prospección, se logró extraer un fragmento de húmero, escápulas, un atlas y un fragmento de defensa. Se determinó que estos restos pertenecen a Mammuthus imperator (LagunasRodríguez y Suárez-Cruz, 1997), especie ahora sinonimizada con Mammuthus columbi (Agenbroad, 2005).También a finales de la década de los noventa, el doctor Mario Pichardo publicó una serie de artículos en los que revisó los trabajos bioestratigráficos de la Fundación Alemana para la Investigación Científica. En su revisión, el doctor Pichardo notó que las dataciones de radiocarbono hechas por el equipo alemán proporcionaban fechas muy similares a las del equipo estadounidense liderado por la doctora Irwin-Williams, quien curiosamente nunca tomó en

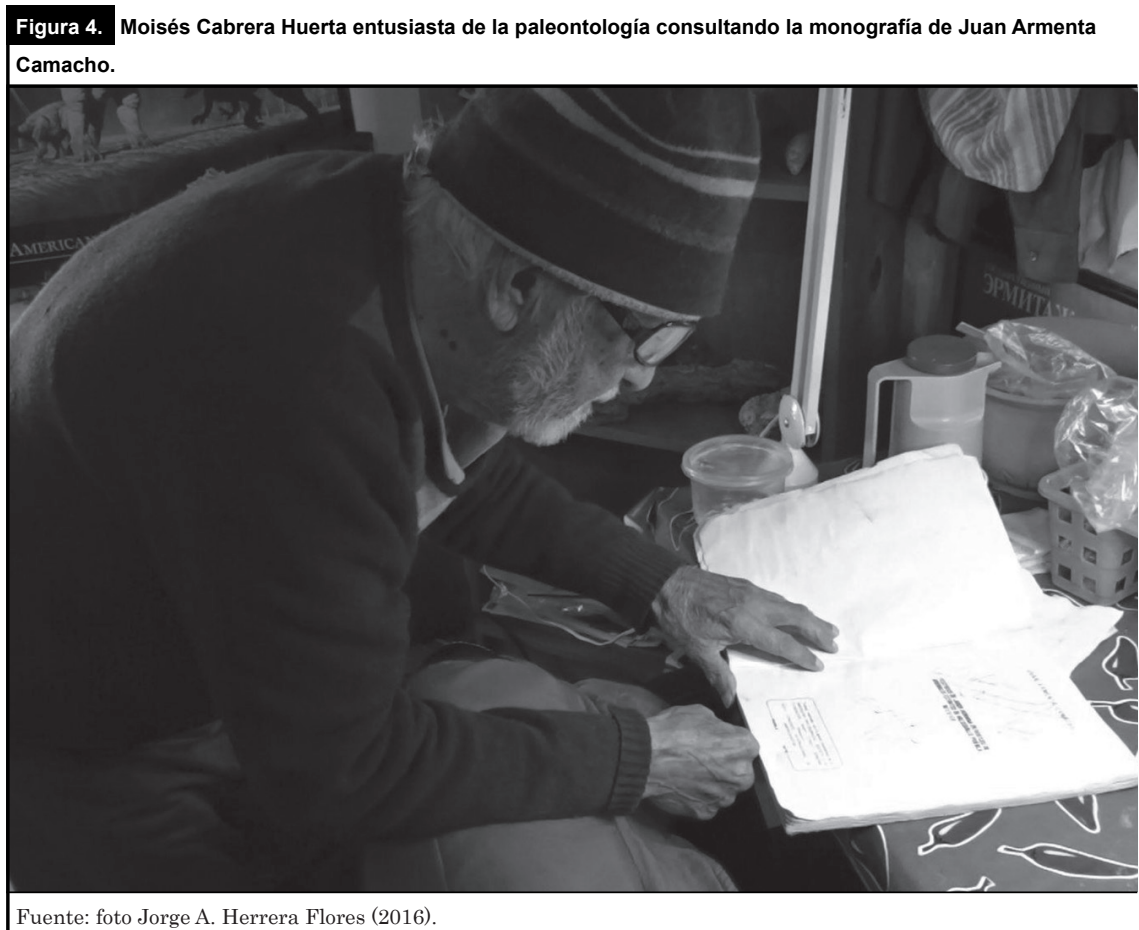


cuenta los trabajos de los investigadores alemanes para corroborar sus datos. En sus trabajos, Pichardo (1997, 1999, 2000, 2001) ofrece una revisión taxonómica y una lista actualizada de la megafauna de Valsequillo. Se enfoca sobre todo en las especies de bisontes, équidos y proboscídeos, así como en su correlación estratigráfica con otros sitios pleistocénicos similares.

Por otra parte, en 1995 un grupo de investigadores de la Universidad Autónoma del Estado de Hidalgo (Castillo-Cerón et al., 1998) retomó las investigaciones en el área de Valsequillo a través del proyecto Correlación bioestratigráfica de las localidades con vertebrados pleistocénicos de los estados de Hidalgo, Puebla y Tlaxcala. Este proyecto incluyó la revisión del material colectado por el Proyecto Valsequillo depositado en la colección del INAHPuebla, el cual se encontraba abandonado desde hacía años sin que se supiera con cuantos especímenes se contaba y a qué especies pertenecían (CastilloCerón et al., 1998; Cruz-Muñoz et al., 1998; Cruz-Muñoz, 2001; Rojas-Cortés, 2004, 2016). La revisión del material del Proyecto Valsequillo consistió en la catalogación y clasificación taxonómica de los fósiles, trabajo que llevó a cabo la biología Valeria Cruz Muñoz como parte de su tesis de licenciatura (CruzMuñoz et al., 1998; Cruz-Muñoz, 2001; Rojas-Cortés, 2004, 2016).

El trabajo de Cruz-Muñoz (2001) dio como resultado la curación y catalogación de importante material fósil. Se clasificaron 2253 especímenes pertenecientes a 15 especies, repartidas en siete órdenes: Testudines (tortugas), Carnivora (cánidos, félidos, mustélidos), Proboscidea (mamuts y mastodontes), Perissodactyla (équidos y tapires), Artiodactyla (bóvidos, camélidos, antilocápridos, tayasuidos), Cingulata (gliptodontes) y Pilosa (perezosos gigantes). El trabajo de Cruz-Muñoz
(2001) también permitió realizar varios estudios posteriores como la descripción de los fósiles de los tayasuidos del género Platygonus (Rojas-Cortés, 2004), la documentación de nuevos registros y ampliación del rango de distribución geográfico de los micromamíferos de las especies Baiomys musculus, Cratogeomys castanops, Cratogeomys merriami, Cynomys mexicanus, Hodomys alleni, Lepus callotis, Microtus mexicanus, Neotoma mexicana, Peromyscus maldonadoi, Reithrodontomys megalotis, Romerolagus diazi, Sigmodon bispidus, Sylvilagus audubonii, Thomomys umbrinus (Cruz-Muñoz, 2006), así como estudios de la dinámica poblacional del caballo mexicano Equus conversidens (Robles-Romero, 2006) y un nuevo registro para las tortugas del género Trachemys (Herrera-Flores, 2009). Hasta el día de hoy, la colección de fósiles del INAH-Puebla sigue siendo consultada por paleontólogos mexicanos, ya que provee información valiosa sobre la prehistoria del Valle de Puebla.

\section{Prospectiva}

La paleontología como ciencia ha tenido grandes avances durante las últimas décadas debido a la aparición de nuevos métodos que permiten explorar con mayor detalle aspectos sobre la biología y ecología de la extinta flora y fauna que hasta hace poco eran muy difíciles de conocer. Un ejemplo de esto es el uso de isotopos estables para inferir las dietas de los mamíferos fósiles del Pleistoceno, lo cual nos deja ver más allá de lo que nos dicen los huesos. En el caso de México, y en específico del Valle de Puebla, estos estudios se han empleado desde hace relativamente poco tiempo; sin embargo, los resultados obtenidos proporcionan datos relevantes sobre el tipo de vegetación que existió en la zona. Pérez-Crespo et al. (2015) en un estudio reciente señalaron que caballos, mamuts y perezosos mostraban una dieta basada en plantas $\mathrm{C}_{4}$, mientras que los carpinchos y gonfoterios poseían una dieta mixta $\mathrm{C}_{3} / \mathrm{C}_{4}$. Se sugiere entonces que la vegetación en Valsequillo era abierta y de poca cobertura arbórea. Otro uso destacable del uso de isotopos estables es poder reconstruir el tipo de clima que existió en cierta zona durante la prehistoria. Por ejemplo, Stevens et al. (2012) llevaron a cabo un estudio en el área de Valsequillo mediante el análisis de caracoles terrestres y marinos a partir del cual determinaron que el clima de la zona hace 35000 años era bastante parecido al clima actual. No obstante, hace 35000 y 20000 años este se volvió considerablemente más seco y con posterioridad más húmedo. Sin lugar a dudas, estos nuevos estudios basados en marcadores biogeoquímicos han significado un gran avance respecto a los anteriores estudios que sólo se limitaban a la extracción y descripción taxonómica de los fósiles. Esto no significa que la paleontología tradicional deje de tener importancia, sino que por el contrario los estudios descriptivos de fósiles ahora pueden ser complementados con análisis más complejos como los mencionados, lo que a su vez nos proporciona una perspectiva más amplia de cómo fue el ambiente específico que existió en determinada zona durante la prehistoria. $\mathrm{Al}$ respecto, la inclusión de nuevos métodos dentro de la paleontología es un aliciente para mantener el interés del público en general en el estudio de la flora y fauna que existió en sus regiones durante el pasado lejano. Es de resaltar que si para el público no especialista es de por sí atractivo el estudio de los fósiles, la inclusión de métodos y análisis novedosos serán sin duda un gran atractivo para que los jóvenes se sientan aún más interesados en la ciencia o incluso se planteen un futuro académico dentro de la paleontología. 


\section{Conclusiones}

Como se puede apreciar, la historia de los estudios paleontológicos del Valle de Puebla es larga, en ocasiones complicada, pero sin lugar a dudas muy productiva. Especialmente la zona de Valsequillo es destacable gracias a que en ella son comunes importantes descubrimientos, algunos de ellos de gran repercusión internacional. Para

Agenbroad, L. D. (2005). North American Proboscideans: Mammoths: The state of knowledge. Quaternary International, 126, 73-92.

Anónimo (1960). Extraordinarios hallazgos prehistóricos en el estado (1960, 12 de enero). El Sol de Puebla (pp. 1, 3).

Armenta, J. (1959). Hallazgo de un artefacto asociado con mamut, en el Valle de Puebla. México: Instituto Poblano de Antropología e Historia e Instituto Nacional de Antropología e Historia.

Armenta-Camacho, J. (1978). Vestigios de labor bumana en buesos de animales extintos de Valsequillo, Puebla, México. Puebla: Consejo Editorial del Gobierno del Estado de Puebla.

Bunde, H. (1973). Geologische Untersuchungen im Gebiet des Valsequillo südlich von Puebla, en W. Lauer (ed). Das Mexikoprojekt der Deutschen ForschungsgemeinschaftBand VI Geologische und paläontologische untersuchungen im Valsequillo bei Puebla (Mexiko) (pp. 21-93). Franz Steiner Verlage GMBH-Wiesbaden.

Castillo-Cerón, J. M., Cabral-Perdomo, M. A., Castro-Azuara, H., Cruz-Muñoz, V., Robles-Romero, O. y Lagunas-Gutiérrez, Z. (1998). Vertebrados fósiles del área de Valsequillo, Puebla, en Memorias del fortuna de los paleontólogos, la riqueza fosilífera del Valle de Puebla sigue proporcionando grandes cantidades de fósiles, por lo que las prospecciones continúan. A diferencia del pasado ahora se emplean métodos de colecta y controles estratigráficos más estrictos. Con esto en un futuro cercano se espera conocer más sobre la diversidad biológica que había en el Valle de Puebla hace miles de años atrás. También es digno de destacar que las investigaciones pasadas y presentes son fuente de inspiración para jóvenes que desean saber más sobre la fauna prehistórica que habitó en su región y los motiva a seguir una carrera dentro de la paleontología. Por este motivo es necesario que este tipo de investigaciones continúen y que además los investigadores ayuden a difundir sus trabajos entre el público en general.

Referencias

VI Congreso Nacional de Paleontología. (pp. 16-17). México: Sociedad Mexicana de Paleontología

Cruz-Muñoz, V. (2001). Catálogo de vertebrados fósiles del Centro Regional INAH de Puebla (tesis de licenciatura). México: Benemérita Universidad Autónoma de Puebla.

Cruz-Muñoz, V. (2006). Micromamiferos del

Pleistoceno tardio de Valsequillo, Puebla, México (tesis de maestría). México: Universidad Nacional Autónoma de México.

Cruz-Muñoz, V., Castro-Azuara, H. E. M., Rojas-Cortés, F. y Robles-Romero, O. J. (1998). Catálogo Paleofaunístico del Centro Regional INAH de Puebla, en Memorias del IV Congreso Nacional de Mastozoología (pp. C05). Xalapa: Asociación Mexicana de Mastozoología A. C.

Freudenberg, W. (1910). Die Säugetierfauna des Pliocäns und Postpliocäns von Mexiko. I. Carnivoren. Geologische und Paläontologische Abhandlungen, 9(3), 195-231.

Freudenberg, W. (1922). Die Säugetierfauna des Pliocäns und Postpliocäns von Mexiko. II. Tiel; Mastodonten und Elefanten. Geologische und Paläontologische Abhandlungen, 14(3), 103-176.

Guenther, E. W. (1967). Ausgrabungen einer eiszeitlichen Tierwelt im Valsequillo (Hochland von Mexiko). Quartär, 163-172.
Guenther, E. W. (1968). Untersuchungen zur jungeiszeitlichen und nacheiszeitlichen geologischen und paläontologischen Geschichte, In F. Tichy (ed.), Das Mexikoprojekt der Deutschen ForschungsgemeinschaftBand I Berichte über begonnene und geplante arbeiten (pp.32-37). Franz Steiner Verlage GMBH-Wiesbaden.

Guenther, E. W. (1973a). Einführung in die geologischen und paläontologischen Untersuchungen, In W. Lauer (ed.), Das Mexiko-projekt der Deutschen Forschungsgemeinschaft-Band VI Geologische und paläontologische untersuchungen im Valsequillo bei Puebla (Mexiko) (pp. 1-20). Franz Steiner Verlage GMBH-Wiesbaden.

Guenther, E. W. (1973b). Elefantenbackenzähne aus dem Valsequillo südlich von Puebla, In W. Lauer (ed.), Das Mexikoprojekt der Deutschen ForschungsgemeinschaftBand VI Geologische und paläontologische untersuchungen im Valsequillo bei Puebla (Mexiko) (pp. 109-177). Franz Steiner Verlage GMBH-Wiesbaden.

Guenther, E. W. y Bunde, H. (1973). Investigaciones geológicas y paleontológicas en México durante los años de 1965 a 1969. Comunicaciones Proyecto Puebla-Tlaxcala, 7,19-20. 
Herrera-Flores, J. A. (2009). Restos fósiles de tortugas en San Buenaventura Nealtican, Puebla. Acta Zoológica Mexicana, 25(3), 455-464.

Herrera-Flores, J. A. (2015). El Museo de Historia Natural de Puebla (1977-2002). Elementos, 98, 43-48.

Irwin-Williams, C. (1962). Preliminary report on investigations in the region of the Valsequillo reservoir 1962. Reporte del Departamento de Prehistoria del Instituto Nacional de Antropología e Historia. México.

Irwin-Williams, C. (1964). Preliminary report on investigations in the region of the Valsequillo reservoir 1964. Reporte del Departamento de Prehistoria del Instituto Nacional de Antropología e Historia. México.

Irwin-Williams, C. (1966). Preliminary report on investigations in the region of the Valsequillo reservoir 1966. Reporte del Departamento de Prehistoria del Instituto Nacional de Antropología e Historia. México.

Irwin-Williams, C. (1967). Associations of early man with horse, camel, and mastodon at Hueyatlaco, Valsequillo (Puebla, Mexico), en P. S. Martin y H. E. Wright (eds.), Pleistocene extinctions: the search for a cause (pp. 337-347). USA: Yale University Press, New Heaven.

Irwin-Williams, C. (1969). Comments on the associations of archaeological materials and extinct fauna in the Valsequillo Region, Puebla, Mexico. American Antiquity, 34(1), 82-83

Irwin-Williams, C. (1978). Summary of archaeological evidence from the Valsequillo region, Puebla, Mexico, en D. L. Browman (ed.), Cultural continuity in Mesoamerica (pp. 7-22). Mouton Publishers.

Irwin-Williams, C. (1981). Commentary on geologic evidence for age of deposits at Hueyatlaco archaeological site, Valsequillo, Mexico. Quaternary Research, 16(2), 258.
Irwin-Williams, C. y Armenta-Camacho, J. (1963). Explorations and excavations near Valsequillo, Mexico. The American Philosophical Society, Year Book. 1963, 550-553.

Lagunas-Rodríguez, Z. y Suárez-Cruz, S. (1997). Los restos de mamut encontrados en Tres Cerritos, Puebla, Puebla. Revista Mexicana de Estudios Antropológicos, 43, 91-108.

Lorenzo, J. L. (1967). Sobre método arqueológico. Boletín INAH. México: Instituto Nacional de Antropología e Historia.

Miller, W. E. y Carranza-Castañeda, O. (1984). Late Cenozoic mammals from central Mexico. Journal of Vertebrate Paleontology, 4(2), 216-236.

Nobis, G. (1973). Die Equidenrestre aus dem Pleistozän des Valsequillo, In Lauer, W. (ed.), Das Mexiko-projekt der Deutschen Forschungsgemeinschaft-Band VI Geologische und paläontologische untersuchungen im Valsequillo bei Puebla (Mexiko) (pp. 95-108). Franz Steiner Verlage GmBH-Wiesbaden.

Osborn, H. F. (1905). Recent vertebrate paleontology. Fossil mammals of Mexico. Science, 21(546), 931-932.

Pérez-Crespo, V. A., Arroyo-Cabrales, J., Alva-Valdivia, L. M., Morales-Puente, P., Cienfuegos-Alvarado, E., Otero, F. J. y Ochoa-Castillo, P. (2015). La paleodieta de cinco especies de mamíferos herbívoros rancholabreanos de Valsequillo (Puebla, México). Revista Chilena de Antropología, 76-82.

Pichardo del Barrio, M. (1960). Proboscídeos fósiles de México: una revisión. Serie Investigaciones 4 . Instituto Nacional de Antropología e Historia.

Pichardo, M. (1997). Valsequillo biostratigraphy: new evidence for Pre-Clovis date. Anthropologischer Anzeiger, 233-246.

Pichardo, M. (1999). Valsequillo biostratigraphy II. Bison, tools, correlate with Tequixquiac. Antbropologischer Anzeiger, 13-24.
Pichardo, M. (2000). Valsequillo biostratigraphy III: Equid ecospecies in paleoindian sites. Anthropologischer Anzeiger, 275-298.

Pichardo, M. (2001). Valsequillo Biostratigraphy IV: Proboscidean Ecospecies in Paleoindian Sites. Anthropologischer Anzeiger, 41-60.

Robles-Romero, O. J. (2006). Dinámica poblacional de Equus conversidens (Perissodactyla: Equidae) del Pleistoceno de Valsequillo, Puebla (tesis de licenciatura). México: Benemérita Universidad Autónoma de Puebla.

Rojas-Cortés, M. F. (2004). Tayassuidos pleistocénicos del área de Valsequillo, Puebla, México (tesis de licenciatura). México: Benemérita Universidad Autónoma de Puebla.

Rojas-Cortés, M. F. (2016). Los mamíferos fósiles de Valsequillo. Saberes y Ciencias, 48, 8.

Steen-McIntyre, V., Fryxell, R. y Malde, H. E. (1981). Geologic evidence for age of deposits at Hueyatlaco archeological site, Valsequillo, Mexico. Quaternary Research, 16(1), 1-17.

Stevens, R. E., Metcalfe, S. E., Leng, M. J., Lamb, A. L., Sloane, H. J., Naranjo, E. y González, S. (2012). Reconstruction of late Pleistocene climate in the Valsequillo Basin (Central Mexico) through isotopic analysis of terrestrial and freshwater snails. Palaeogeography, Palaeoclimatology, Palaeoecology, $319,16-27$

Szabo, B. J., Malde, H. E. y Irwin-Williams, C. (1969). Dilemma posed by uranium-series dates on archaeologically significant bones from Valsequillo, Puebla, Mexico. Earth and Planetary Science Letters, 6, 237-244.

Treue, W. (1968). Das Mexico-Projekt. Ein Unternehmen deutsch-mexikanischer interdisziplinärer Regionalforschung, In F. Tichy (ed.), Das Mexiko-projekt der Deutschen Forschungsgemeinschaft-Band I Berichte über begonnene und geplante arbeiten. Franz Steiner Verlage GMBH-Wiesbaden. 